\title{
Índice de Sustentabilidade do Monumento Natural da Gruta do Lago Azul, Bonito, Mato Grosso do Sul, Brasil
}

\author{
Sustainability Index of the Gruta do Lago Azul Natural Monument, Bonito Mato \\ Grosso do Sul, Brazil
}

\author{
Marta Regina da Silva-Melo ${ }^{1}$ \\ Neiva Maria Robaldo Guedes ${ }^{2}$ \\ Gleidson André Pereira de Meló
}

Palavras-chave:
Patrimônio Natural
Grutas Turísticas
Barômetro da Sustentabilidade
Turística

\begin{abstract}
Resumo
Com relevante beleza cênica, a Gruta do Lago Azul é uma das mais importantes cavernas do patrimônio espeleológico nacional e um atrativo turístico com visibilidade mundial. O presente estudo tem como objetivo analisar o índice de sustentabilidade do Monumento Natural da Gruta do Lago Azul, Bonito, Mato Grosso do Sul. Foi utilizado o Barômetro da Sustentabilidade Turística (BST) e o modelo AMOEBA de Indicadores de Sustentabilidade do Turismo (AIST) para analisar o nível de sustentabilidade. Foram selecionados vinte indicadores que resultaram nos seguintes índices: o Sistema Humano apresentou média de $48,62 \%$ e o Sistema Ecológico foi equivalente a 58,17\%. O índice verificado encontra-se em um estágio intermediário, proporcional a 41-60\%, considerado satisfatório. Apesar disso, foi constatada a inexistência de um Plano de Manejo e de um Programa de Educação Ambiental, previstos no Sistema Nacional de Unidade de Conservação. Diante do exposto, infere-se que a integralidade do nível de sustentabilidade do Monumento Natural da Gruta do Lago Azul ainda não acontece de forma efetiva. Portanto, fomentar um autêntico turismo sustentável em áreas que dispõe de serviços ambientais essenciais para a sociedade, como a Gruta do Lago Azul, é implementar um desenvolvimento compatível em atender às necessidades das gerações atuais, sem comprometer a capacidade das gerações futuras.
\end{abstract}

\section{Keywords}

Natural heritage

Tourist cave

Barometer of Tourism

Sustainability

\begin{abstract}
With relevant scenic beauty, the Gruta do Lago Azul is one of the most important caves with national speleological heritage and is a tourist attraction with worldwide visibility. This study aims to analyze the sustainability index of the Natural Monument of the Gruta do Lago Azul in Bonito, Mato Grosso do Sul state. The Barometer of Tourism Sustainability (BTS) and AMOEBA of Tourism Sustainability Indicators (ATSI) were used to analyze the level of sustainability. Twenty indicators were selected that resulted
\end{abstract}

\footnotetext{
${ }^{1}$ Universidade Estadual de Mato Grosso do Sul. Campo Grande, MS. martamelors@gmail.com

${ }^{2}$ Universidade Anhanguera-Uniderp. Campo Grande, MS. guedesneiva@gmail.com

${ }^{3}$ Instituto Federal de Mato Grosso do Sul. Campo Grande, MS. gandmelo@gmail.com
} 
in the following indices: the Human System had an average of $48.62 \%$ and the Ecological System was $58.17 \%$. The verified index is in an intermediate stage, proportional to $41-60 \%$, and is considered satisfactory. Despite this, a Management Plan and an Environmental Education Program were absent, and these are required by the National System of Conservation Units (SNUC). Given the foregoing, it is inferred that the integrity of the level of sustainability of the Natural Monument of the Gruta do Lago Azul is still uncertain. Therefore, to promote authentic sustainable tourism in areas that provide essential environmental services to society, such as the Gruta do Lago Azul, it is necessary to implement a compatible level of development to meet the needs of current generations, without compromising the capacity of future ones.

\section{INTRODUÇÃO}

O turismo em cavidades naturais é caracterizado pela visitação em áreas subterrâneas, e se destaca como atividade que cresce continuamente no mundo todo. Além da importância científica e cultural, as grutas constituem um patrimônio de valor imensurável para a humanidade (LOBO; BOGGIANI, 2013).

As primeiras grutas turísticas surgiram há mais de 400 anos. A caverna Postojna, na Eslovênia, foi a primeira a ser estruturada para visitação turística no ano de 1818, sendo iluminada a partir de 1918 (ARAUJO et al., 2017). Durante o século XX ocorreu um crescimento significativo na demanda por grutas turísticas (CIGNA; FORTI, 2013). Entretanto, esse crescimento não tem sido acompanhado pelo planejamento e manejo adequado dos locais visitados, principalmente em ambientes frágeis como as cavidades naturais (LOBO, 2014).

No Brasil essa prática não é diferente, e, devido às características naturais associadas a paisagens cársticas (MORETTI et al., 2016), e ao grande potencial de cavernas turísticas distribuídas em todo o território nacional, a visitação ocorre há décadas em diferentes estados brasileiros, dentre os quais: São Paulo, Minas Gerais, Rio de Janeiro, Goiás, Bahia e Ceará.

No Mato Grosso do Sul localiza-se Bonito, um destino de turismo cárstico (Lobo; Boggiani, 2013), e dentre seus atrativos turísticos, a Gruta do Lago Azul se sobressai como Monumento Natural de beleza cênica e com características admiráveis.

É um Patrimônio natural e cientifico, relevante em razão de registros biológicos, geológicos, geomorfológicos e paleontológicos (LOBO, 2015). Com visibilidade mundial, constitui um dos atrativos turísticos mais visitados da região (MORETTI et al., 2016).
A gruta apresenta espeleotemas de rara beleza, e, em razão dessas características, é indispensável um planejamento contínuo à atividade de contemplação, haja vista que a prática necessita ser conduzida dentro de bases criteriosas, devido à fragilidade do ecossistema (CORDEIRO et al., 2013). Sobretudo, deve se levar em consideração a necessidade de mitigar fatores como o alto grau de solubilidade da rocha, a fragilidade de suas formações e a extensa rede de drenagem subterrânea (GRECHI et al., 2010).

Pela sua importância, a Gruta do Lago Azul foi tombada em 1978 pelo Instituto do Patrimônio Histórico e Artístico Nacional (IPHAN). Por meio do Decreto Estadual $\mathrm{n}^{\circ}$ 10.394/2001, o governo do Mato Grosso do Sul elevou essa área para Unidade de Conservação na categoria Monumento Natural, que tem como propósito a conservação de sítios naturais raros, singulares ou de grande beleza cênica (BRASIL, 2000), e garantir a integridade das Grutas do Lago Azul e Nossa Senhora Aparecida.

A Gruta do Lago Azul é considerada uma das mais importantes cavernas do patrimônio espeleológico brasileiro e um dos mais notáveis atrativos naturais (BOGIANNI et al., 2009).

Nesse sentido, o turismo contribui para fortalecer a conexão dos visitantes com os valores das áreas naturais protegidas e pode ajudar na sensibilização em relação à conservação do patrimônio que essas áreas envolvem (SALAS et al., 2019). Assim, desenvolver atividades turísticas de forma sustentável implica em ações socialmente justas, economicamente viáveis e ecologicamente corretas.

Desse modo, o levantamento de índices de sustentabilidade pode representar indicativos capazes de mensurar as ações do turismo, e com isso propor medidas mitigadoras dos impactos ambientais e socioculturais negativos, e, de igual forma, assegurar que as atividades desenvolvidas nos espaços naturais sejam planejadas com respaldo nas diretrizes 
ambientais e sociais básicas para a sustentabilidade. Por isso a realização do turismo em espaços naturais protegidos deve conciliar os princípios que respeitem o uso sustentável dos recursos naturais e a dinâmica ambiental necessários à manutenção da sustentabilidade (MATHEUS; RAIMUNDO, 2016).

Assim, a importância desse estudo consiste refletir em uma prática turística sustentável de maneira que garanta, em longo prazo, a gestão efetiva desse atrativo natural, de forma que atenda às necessidades das gerações atuais, sem comprometer a capacidade das gerações futuras. Portanto, o presente estudo tem como objetivo analisar o índice de sustentabilidade do Monumento Natural da Gruta do Lago Azul, Mato Grosso do Sul.

\section{MATERIAL E MÉTODOS}

\section{Área de estudo}

O Monumento Natural Gruta do Lago Azul está localizado no município de Bonito, Mato Grosso do Sul, sob as coordenadas $21^{\circ} 08^{\prime} 41^{\prime \prime} \mathrm{S}$ e $56^{\circ} 35^{\prime} 27^{\prime}$ 'W, entre os córregos Anhumas, ao norte; e Taquaral, ao sul (Figura 1). Um patrimônio relevante que compreende duas áreas não contíguas do complexo de Grutas da Serra da Bodoquena, representadas pelas Grutas do Lago Azul e Nossa Senhora Aparecida. Atualmente, apenas a Gruta do Lago Azul pode ser visitada, e por questões de segurança, a Gruta de Nossa Senhora Aparecida é fechada à visitação (BOGIANNI et al., 2009).

Figura 1. Mapa de situação do Monumento Natural Gruta do Lago Azul, no município de Bonito,

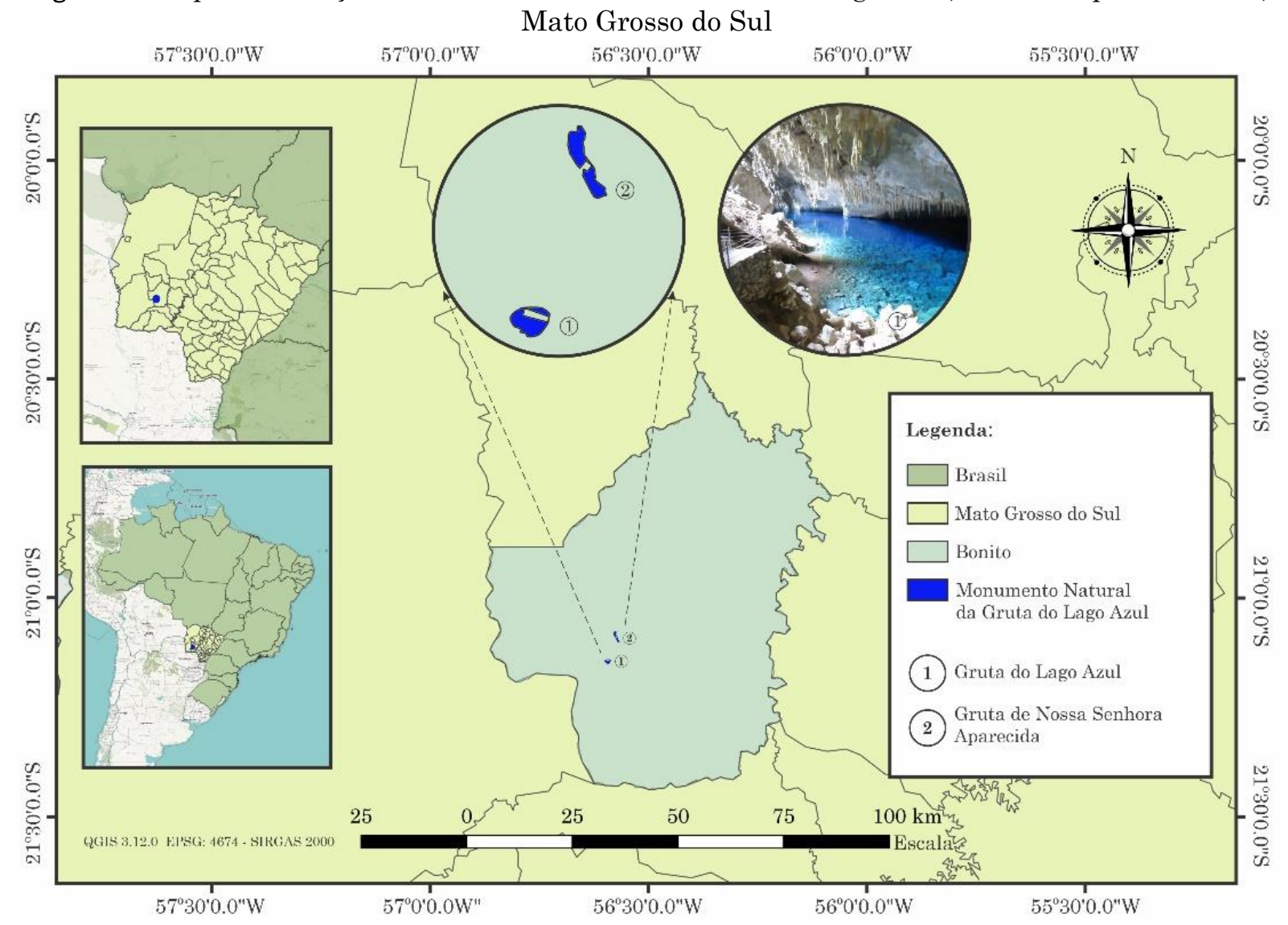

Fonte: organizado pelos autores, 2020.

A Gruta do Lago Azul se destaca por ser uma Unidade de Conservação de significativa notabilidade no cenário turístico nacional e internacional, além de ser um patrimônio natural de grande importância na provisão de serviços ecossistêmicos.

\section{Coleta e análise de dados}

Foram analisados dados coletados referentes aos meses de julho de 2017 a julho 2019, levando em consideração a sazonalidade turística, ou melhores períodos de alta e baixa temporada. Foi aplicado um questionário aos visitantes da Gruta do Lago Azul, autorizado pelo Comitê de 
Ética em Pesquisas da Universidade Anhanguera-Uniderp, sob o Parecer $\mathrm{n}^{\mathrm{o}}$ 2.526.285.

Os métodos utilizados para analisar os dados foram o Barômetro da Sustentabilidade Turística (BST); e o Modelo Amoeba de Indicadores de Sustentabilidade do Turismo (Amoeba of Tourism Sustainability Indicators ATSI), que se caracteriza por ser uma representação gráfica em forma de radar. Ambos os modelos auxiliaram na verificação do nível de sustentabilidade do Monumento Natural da Gruta do Lago Azul, nas dimensões ambiental, econômica e social. No caso, é recomendado que o BST seja utilizado simultaneamente com o modelo Amoeba, visto que, quando associados são capazes de apresentar os indicadores de maneira eficaz (KO, 2005).

O BST foi adaptado do Barômetro da Sustentabilidade (BS), criado por Prescott-Allen, em 1997, com o propósito de mensurar a sustentabilidade global e local, designado a órgãos governamentais e não-governamentais. Essa ferramenta é utilizada com escala que varia de 1 a 100, para facilitar a compreensão da sustentabilidade ambiental e social (Tabela 1).

Tabela 1. Escala dos valores da sustentabilidade para mensurar o nível do bem-estar ecológico e do bem-estar humano

\begin{tabular}{lc}
\hline \multicolumn{1}{c}{ Setor } & Valores da Escala (\%) \\
\hline Sustentável (excelente) & $81-100$ \\
Potencialmente sustentável (bom) & $61-80$ \\
Intermediário & $41-60$ \\
Potencialmente insustentável (pobre) & $21-40$ \\
Insustentável (ruim) & $1-20$ \\
\hline
\end{tabular}

Fonte: elaborado com base em Prescott-Allen (1997).

A utilização do BST e do modelo Amoeba (Figuras 2 e 3) fornecem um panorama geral da área estudada sobre os sistemas humano e ecológico. Além de concentrar informações apresentadas em um gráfico com leitura intuitiva e compreensível. Para a mensuração dos dados foram utilizados parâmetros para cada indicador, com base na escala de Prescott-Allen (1997).

Figura 2. Representação gráfica do Barômetro da Sustentabilidade Turística (BST)

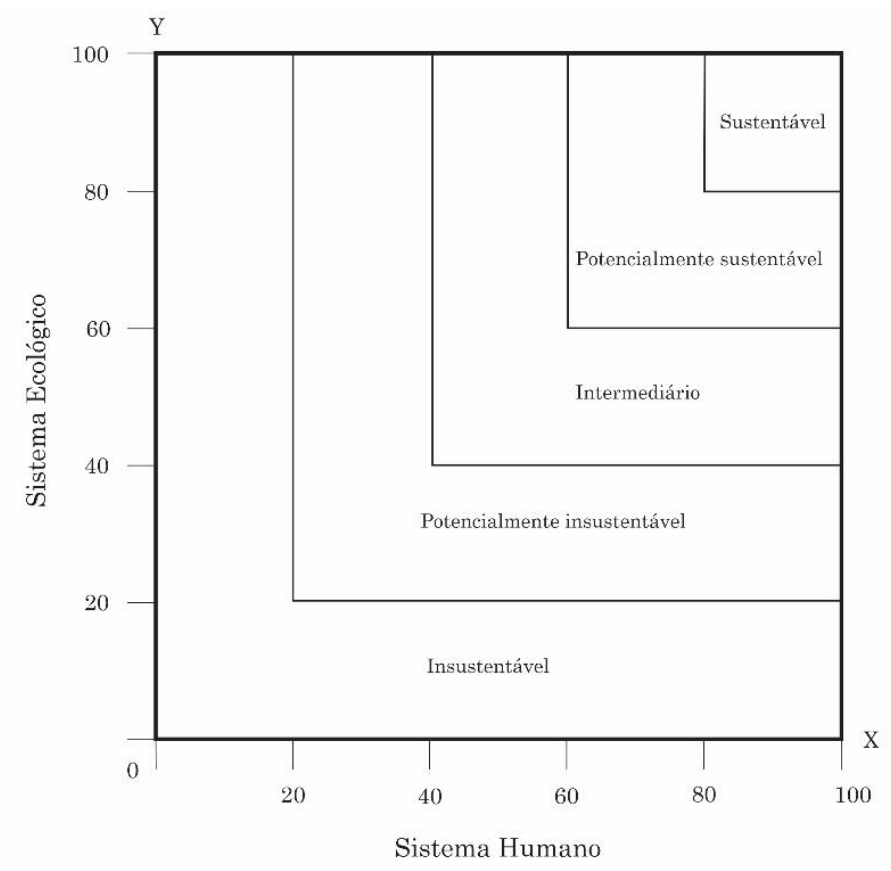

Fonte: elaborado com base em Ko (2005). 
Figura 3. Representação gráfica do modelo Amoeba of Tourism Sustainability Indicators (ATSI)

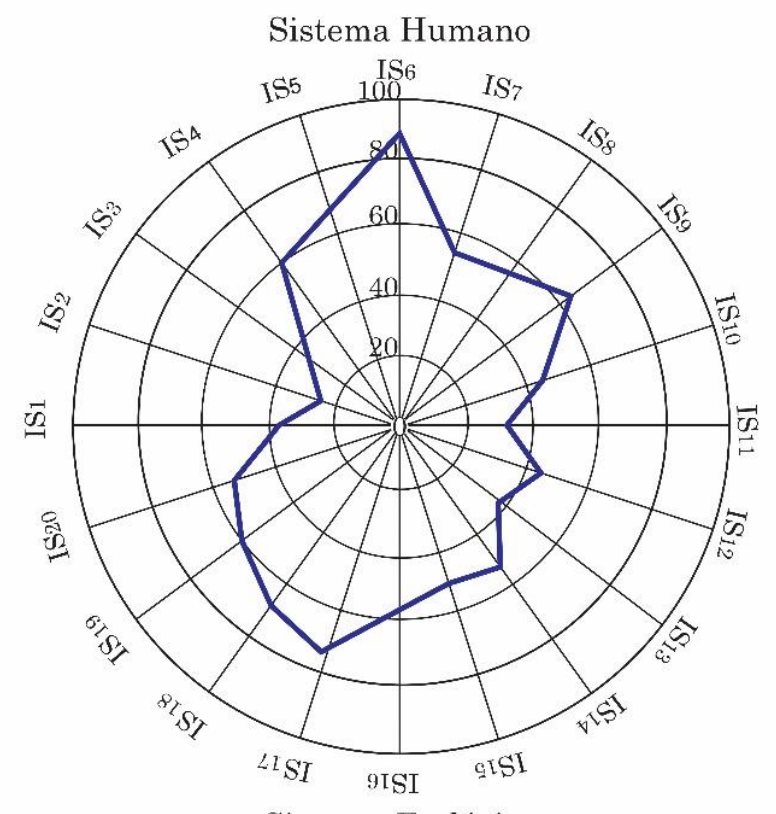

Sistema Ecológico

Fonte: elaborado com base em Ko (2005).

A escolha dos indicadores teve como aporte a aplicação de questionário para uma amostra aleatória $(n=387)$, entrevista com o gestor da área, referencial teórico, análise de documentos e visitas in loco.

Foi utilizado para a verificação do grau de confiabilidade dos dados coletados nos questionários, o teste estatístico Alpha de Cronbach, conforme a equação (1). O coeficiente é sinalizado como forma de estimar a confiabilidade de um questionário aplicado em uma pesquisa (HORA et al., 2010).

$$
a=\left[\frac{k}{k-1}\right] X\left[\begin{array}{c}
k \\
\sum S_{1}^{2} \\
S_{t}^{2}
\end{array}\right]
$$

Onde:

k corresponde ao número de itens do questionário;
$S_{i}^{2}$ corresponde a variância de cada item; e $\boldsymbol{S}_{\boldsymbol{t}}^{2}$ corresponde a variância total do questionário, correspondente à soma de todas as variâncias.

A consistência dos dados analisados corresponde ao Alpha de Cronbach ( $\alpha$ ), com grau de significância, conforme a tabela 2 .

Tabela 2. Escala de valores, segundo o coeficiente Alpha de Cronbach.

\begin{tabular}{cc}
\hline Alpha de Cronbach & Consistência interna \\
\hline$\alpha>0,9$ & Excelente \\
$\alpha>0,8$ & Bom \\
$\alpha>0,7$ & Aceitável \\
$\alpha>0,6$ & Questionável \\
$\alpha>0,5$ & Pobre \\
$\alpha<0,5$ & Inaceitável \\
\hline
\end{tabular}

Fonte: elaborado segundo George e Mallery (2003). 
A credibilidade do instrumento utilizado na verificação do índice de sustentabilidade da Gruta do Lago Azul, mediante o alpha de
Cronbach, confirmou que a consistência do questionário foi significativa (Tabela 3).

Tabela 3. Representa consistência interna do questionário segundo o coeficiente Alpha de Cronbach

\begin{tabular}{ccccc}
\hline Respondentes & $\mathbf{K}$ & $\boldsymbol{S}_{\boldsymbol{i}}^{\mathbf{2}}$ & $\boldsymbol{S}_{\boldsymbol{t}}^{\mathbf{2}}$ & $\mathbf{\alpha}$ \\
\hline $\mathbf{3 8 7}$ & 16 & 17,85 & 92,39 & 0,86 \\
\hline
\end{tabular}

Fonte: organizado pelos autores, conforme os dados da pesquisa.

A validação desse instrumento foi confirmada no valor obtido mediante o alpha de Cronbach ( $\mathbf{a}$ $=0,86$ ).

\section{RESULTADOS E DISCUSSÃO}

Ao considerar que os indicadores são aspectos mensuráveis, os quais possibilitam uma leitura visual dos resultados e diagnóstico para elaboração de programas e projetos (OMT, 2005; VAN BELLEN, 2006), foram selecionados indicadores dos sistemas humano e ecológico que representassem o nível de sustentabilidade da Gruta do Lago Azul (Tabela 4).

Tabela 4. Indicadores selecionados para quantificar o nível de sustentabilidade da Gruta do Lago Azul, Bonito, Mato Grosso do Sul

\begin{tabular}{|c|c|c|c|}
\hline \multirow{11}{*}{ 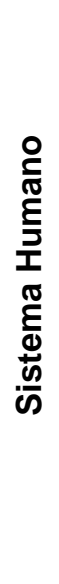 } & Indicadores & IS* & Valores (\%) \\
\hline & Acesso (entre a cidade e o atrativo) & IS1 & 50,50 \\
\hline & Atendimento e receptividade (guias, monitores) & IS2 & 76,70 \\
\hline & Equipamentos de segurança & IS3 & 57,40 \\
\hline & Informação e divulgação & IS4 & 57,10 \\
\hline & Acessibilidade & IS5 & 41,80 \\
\hline & Infraestrutura interna (escadas, corrimãos, passarelas) & IS6 & 46,20 \\
\hline & Difusão da Educação Ambiental & IS7 & 56,50 \\
\hline & Atenção aos atrativos turísticos naturais & IS8 & 72,90 \\
\hline & Gestão de riscos (EPIs, primeiros socorros, condutor experiente) & IS9 & 27,10 \\
\hline & Realização de atividades socioambientais & IS10 & 0,00 \\
\hline \multirow{10}{*}{ 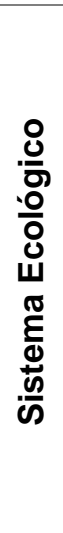 } & Acondicionamento e organização do lixo & IS11 & 70,20 \\
\hline & Vegetação (estado geral de conservação) & IS12 & 65,90 \\
\hline & Visitação pode afetar o espaço natural & IS13 & 43,30 \\
\hline & Desequilíbrio sobre a fauna local & IS14 & 42,80 \\
\hline & Desgaste do solo & IS15 & 66,70 \\
\hline & A gruta constitui ambiente frágil & IS16 & 76,50 \\
\hline & Representa um ecossistema vulnerável & IS17 & 54,40 \\
\hline & Possui informações geológicas relevantes & IS18 & 61,90 \\
\hline & Controle da Capacidade de Carga & IS19 & 100,00 \\
\hline & Plano de Manejo & IS20 & 0,00 \\
\hline
\end{tabular}

IS $^{*}=$ Indicador de sustentabilidade. Fonte: elaborado pelos autores, conforme os dados da pesquisa.

Nível de sustentabilidade da Gruta do Lago Azul

Com base nos 20 indicadores selecionados foi possível extrair a média que originou uma pontuação final, indicando o nível de sustentabilidade da Gruta do Lago Azul. Para o
Sistema Humano a média foi equivalente a 48,62\%; e para o Sistema Ecológico, a média foi equivalente a $58,17 \%$. Tais resultados foram inseridos no BST e no modelo AMOEBA (Figuras 4 e 5). 
Figura 4. Representa o nível de sustentabilidade da Gruta do Lago Azul, segundo o Barômetro da Sustentabilidade Turística

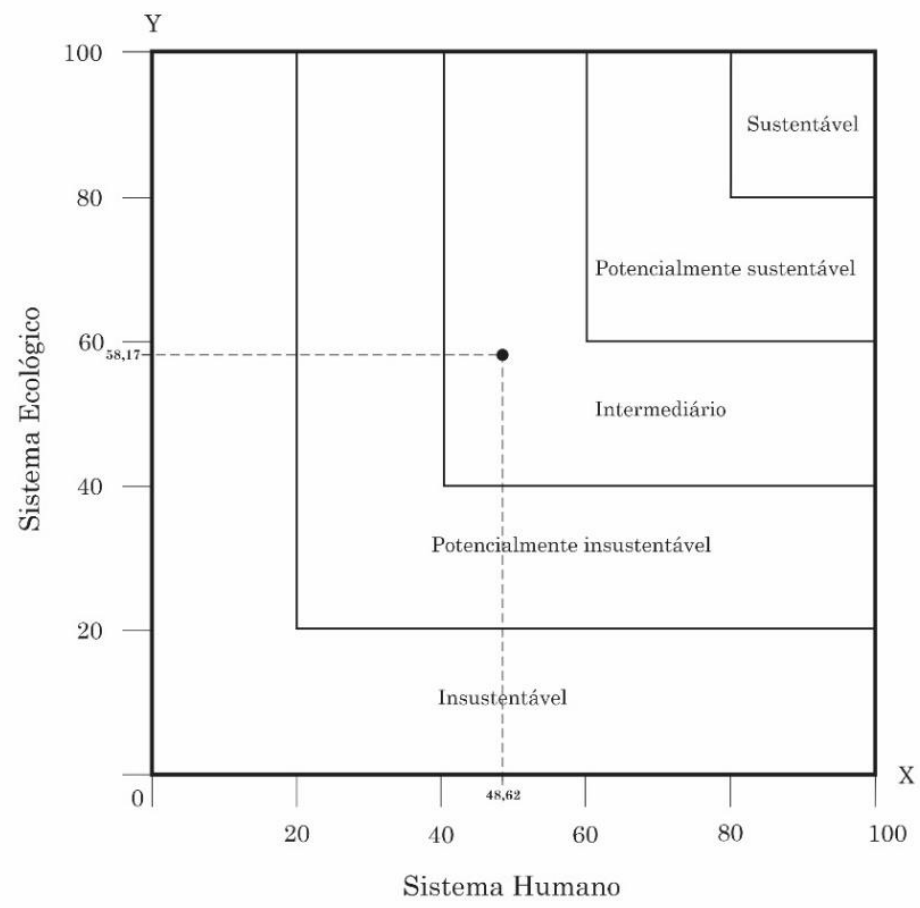

Fonte: Elaborado pelos autores, conforme os dados da pesquisa.

De acordo com a representação gráfica apresentada no BST, foi possível observar que o nível de sustentabilidade do Monumento Natural Gruta do Lago Azul apresentou um estágio intermediário, proporcional a $41-60 \%$. No contexto, o indicativo apresentado é considerado satisfatório na escala de valores da sustentabilidade (PRESCOTT-ALLEN, 1997).

Apesar disso, a evidência dessa informação pode ser um sinal de alerta, resultado que demonstra a necessidade dos gestores reconsiderarem algumas variáveis nos aspectos ambientais e sociais.

Figura 5. Mensuração do nível de sustentabilidade de cada indicador aplicado à Gruta do Lago Azul, conforme modelo AMOEBA

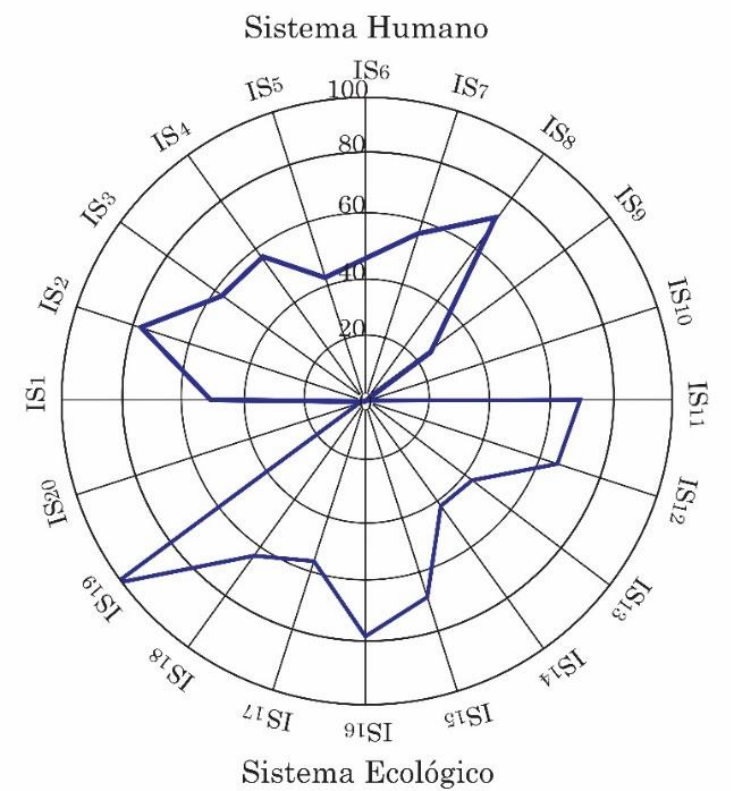

Fonte: Elaborado pelos autores, conforme os dados da pesquisa. 
Entretanto, quando se trata de uma Unidade de Conservação de Proteção Integral, que não apresentou indicadores fundamentais $\mathrm{e}$ compatíveis com os objetivos de sua criação, demonstra preocupação. Visto que, foi constatada a inexistência de um Plano de Manejo, principal instrumento técnico de planejamento e gestão, que tem como objetivo de regular o uso da área e o manejo dos recursos naturais, e propiciar a integração da UC à vida econômica e social das comunidades vizinhas.

O manejo e a gestão de áreas protegidas, não apenas envolvem fatores biofísicos, culturais e socioeconômicos, mas, também, numerosas partes interessadas (HOCKINGS et al., 2019), fatores que apontam as condições e o modelo da gestão.

Teles e Nogueira (2016) mencionam que a maioria das áreas protegidas no Brasil foi criada por Decreto governamental, e muitas dessas áreas não possuem o Plano de Manejo. A Gruta do Lago Azul se inclui nessa situação, pois, desde o ano de 1984 foi realizado um projeto intitulado Projeto Grutas de Bonito, coordenado pelo arquiteto Clayton Ferreira Lino e equipe técnica multi e interdisciplinar, com diretrizes para elaboração de um Plano de Manejo turístico da região (BOGGIANI et al., 2009).

Isso também se confirma no edital de chamamento público do IMASUL ${ }^{\circ}$ 001/2019 processo $n^{\circ} 71 / 403315 / 2018$, para a seleção de entidades sem fins lucrativos, para a elaboração do Plano de Manejo do Monumento Natural da Gruta do Lago Azul, publicado em março de 2019, no Diário Oficial do Mato Grosso do Sul.

A ausência desse documento técnico é manifestada, também, nos dados disponíveis na Plataforma do Cadastro Nacional de Unidade de Conservação (CNUC, 2020). Bem como, na ação judicial do Ministério Público Estadual, em 2018, que ingressou uma Ação Civil de Improbidade Administrativa referente à Obrigação de Fazer Ambiental, do Instituto do Meio Ambiente de Mato Grosso do Sul, Prefeitura Municipal de Bonito e seus respectivos representantes, devido a irregularidades na conservação e prestação de contas do Monumento Natural da Gruta do Lago Azul.

Nesse caso, o Monumento Natural Gruta do Lago Azul, como UC de referência no Brasil, está em déficit em relação ao SNUC, visto que, foi estabelecido que, após a criação de uma Unidade de Conservação, o Plano de Manejo deve ser elaborado em um prazo máximo de cinco anos (BRASIL, 2000).

Enfatiza-se que a elaboração do Plano de Manejo deve seguir orientações do Estudo de Impacto Ambiental, Relatório de Impacto Ambiental, bem como as Diretrizes e Orientações
Técnicas do Planos de Manejo Espeleológico que tratam dos limites temáticos e conteúdos mínimos do Plano de Manejo da Unidade de Conservação (BOGIANNI, 2007).

No aspecto da conservação ambiental, a área tem passado por diferentes transformações para atender à demanda turística. Segundo estudiosos da área, o trecho visitado da Gruta do Lago azul passa por intensas modificações, nas quais conduzem a diversos impactos negativos; e no âmbito da espeleologia, a questão de monitoramento e licenciamento ambiental é incipiente, de modo igual a outros estados brasileiros (CORDEIRO et al., 2013). No caso, é notório que as UCs em diferentes partes do território nacional geram um rearranjo de forças e ações no território em que se implementam (TOFETI; CAMPOS, 2019).

Essa situação também ocorre em outros países, como Portugal, que em alguns casos não realizam qualquer avaliação dos impactos causados pelo fluxo constante de pessoas no ambiente cavernícola (ARAUJO et al., 2017). No Equador, por falta de monitoramento, as cavidades já exploradas e com algum tipo de uso (principalmente em atividades turísticas), apresentam uma degradação acentuada (SÁNCHEZ-CORTEZ et al., 2017).

No caso da Gruta do Lago Azul, não foi observado esse tipo de degradação. Mas, independente dos fatores, se faz necessário monitoramento e manejo que possam minimizar os efeitos negativos e maximizar os benefícios dessa área em longo prazo, a fim de evitar uma marcha apenas no rumo da mercantilização da gruta.

Foi identificado que não existe um programa de atividades socioambientais com ações de educação ambiental que atendam gratuitamente os moradores do município de Bonito e de outros municípios de entorno, capaz de contribuir na formação de cidadãos comprometidos com a conservação ambiental. Necessidade sinalizada por Bogianni e Clemente (1999), sobre a gratuidade para integrantes da comunidade. No entanto, considera-se que é preciso ampliar esse atendimento com oportunidades inclusivas destinadas à visitação ao Monumento Natural.

Oportunamente, é pertinente a implantação de um programa com atividades que integram ações educativas e inclua alunos de escolas da rede pública e privada e a população residente de modo geral. Nesse caso, recomenda-se o uso das diretrizes da Estratégia Nacional de Comunicação e Educação Ambiental (ENCEA) no âmbito do SNUC (MAMEDE, 2008).

Este deve ser posto em prática continuamente para que o processo de Educação Ambiental alcance integralmente todos os atores sociais, 
sejam eles do poder público, iniciativa privada e a sociedade civil organizada, envolvidos na prática turística que incluem as instituições, as agências de viagens, os guias de turismo, monitores, comunidade, visitantes e turistas de modo geral. Pois, há algum tempo são apontadas falhas nos processos de educação ambiental em atrativos como a Gruta do Lago Azul (KLEIN et al., 2011).

Assim, os critérios desse encadeamento podem viabilizar a formação de cidadãos reflexivos sobre as questões ambientais que consideram a conservação e a sustentabilidade como componentes indispensáveis nessa construção.

Face a isso, implementar um programa de educação ambiental é uma ação necessária na visitação de grutas turísticas, pois, a interpretação é um meio de educação informal baseado na experiência em sua totalidade que pode tornar o conhecimento acessível ao público leigo sobre características ambientais da gruta (ARAUJO, 2019). Haja vista que os benefícios advindos pela presença da Unidade de Conservação devem ser compartilhados pelos atores sociais presentes no território.

Nessa situação, a inclusão social nas atividades turísticas pode contribuir para o desenvolvimento sustentável, pois, agrega um conjunto de dimensões favoráveis à solidariedade e à integração social (RUA, 2006). $\mathrm{O}$ acesso em áreas naturais protegidas necessita ser democratizado de forma a não serem interpretados como locais intocáveis da natureza, de uso exclusivo de alguns grupos sociais, e a atividade turística pode ser um dos caminhos (DUTRA, 2017).

Quanto aos indicadores observados, favoráveis à sustentabilidade, foi verificado que no aspecto de arrecadação, essa UC dispõe de provisão satisfatória, por apresentar um dos mais significativos fluxos de visitantes dentre as grutas turísticas do Brasil. Segundo a Secretaria de Turismo de Bonito (2020), a visitação referente aos anos de 2017, 2018 e 2019 apresentaram uma taxa equivalente a 74.501 , 73.810 e 74.794 , respectivamente (OTEB, 2019). Essa condição se diferencia de outras Unidades de Conservação brasileiras, em que poucas conseguem administrar as suas ações (GODOY; LEUZINGER, 2015) de modo sustentável e equilibrado as atividades de turismo dentro e no entorno delas, isso inclui o contexto econômico, ambiental, político e social (TELES; NOGUEIRA, 2016).

Aponta que as especificidades da visitação na Gruta do Lago Azul ocorrem também em detrimento da capacidade de carga da visitação turística da área, estipulado com o número máximo é de 305 visitantes por dia, em grupos de no máximo 15 pessoas, além do guia, com intervalo entre um grupo e outro de no mínimo 20 minutos (BOGIANNI, 2007; KLEIN et al., 2011).

Destaca-se, ainda, como indicador importante para a sustentabilidade, a implantação do voucher único no município de Bonito. O uso desse instrumento possibilitou o controle da capacidade de suporte em atrativos, delimitando o acesso às reservas turísticas, dados estatísticos, controle a arrecadação tributária e a sensibilização do trade turístico acerca da importância dos recursos naturais (GRECHI et al., 2010). Para tanto, considera que a condição financeira, se gerida adequadamente, favorece não só a sustentabilidade econômica da área, mas também os aspectos ambientais e sociais.

A Gruta do Lago Azul além de despontar como instrumento efetivo para a conservação da biodiversidade e proteção das paisagens naturais, propicia às pessoas uma benéfica interação de conhecimento e bem-estar em relação à natureza, valorização da cultura local e oportunidades de aprendizado (LOBO, 2014).

Diante do exposto, enfatiza-se que as políticas de conservação preconizam que os princípios norteadores da sustentabilidade devem compreender integralmente a dimensão ambiental, econômica e social. Quando essas três esferas se entrelaçam de modo coerente se configura o espaço da sustentabilidade, efetivamente (BURSZTYN; BURSZTYN, 2013). Por esse motivo, a visão sobre os benefícios que uma UC oportuniza indicam possibilidades e tendências para a sua gestão.

Por certo, a premissa que rege a sustentabilidade é representada por um desenvolvimento compatível em atender às necessidades das gerações atuais, sem comprometer a capacidade das gerações futuras. Sendo assim, fomentar um autêntico turismo sustentável em áreas que dispõe de serviços ecossistêmicos essenciais à sociedade, é oportunizar para que os benefícios dessa atividade turística sejam compartilhados de forma justa.

\section{CONSIDERAÇÕES FINAIS}

Conclui-se que a integralidade da sustentabilidade turística no Monumento Natural da Gruta do Lago Azul ainda não 
acontece de forma efetiva, pois, os princípios que norteiam a sustentabilidade, que envolvem a dimensão ambiental, social e econômica, não são entrelaçados de modo coerente, e isso pode estar subordinado à configuração de sua gestão.

Apesar dessa área ser reconhecida como uma das mais importantes cavernas do patrimônio espeleológico nacional e um dos mais notáveis atrativos naturais com visibilidade mundial, devido à visitação turística, é indispensável buscar as melhores soluções de sustentabilidade respeitando as dinâmicas ambientais e sociais dessa Unidade de Conservação.

Face a isso, é fundamental que todo o planejamento e manejo seja adequado ao seu uso, principalmente por ser um ambiente frágil e especialmente por proporcionar bem-estar aos seus visitantes e por fortalecer a economia local e regional.

Portanto, sugere-se que na elaboração do Plano de Manejo conste um Programa de Educação Ambiental e integração com as áreas de entorno para uma maior efetividade da sustentabilidade turística. Assim, os critérios desse encadeamento podem viabilizar a formação de cidadãos reflexivos sobre as questões ambientais que consideram a conservação e a sustentabilidade como componentes indispensáveis nessa construção.

\section{AGRADECIMENTOS}

Ao Programa de Pós-Graduação em Meio Ambiente e Desenvolvimento Regional da Anhanguera-Uniderp; à Coordenação de Aperfeiçoamento de Pessoal de Nível Superior (CAPES), pela concessão de bolsa de estudo, modalidade I, código de financiamento 001; ao Instituto de Meio Ambiente do Mato Grosso do Sul; ao gestor do Monumento Natural da Gruta do Lago Azul; e aos entrevistados que participaram voluntariamente da pesquisa.

\section{REFERÊNCIAS}

ARAUJO, H. R. Propostas para o desenvolvimento sustentável do espeleoturismo no Brasil: estudo comparativo com a experiência portuguesa. 2019. 312 f. Tese. Instituto de Geografia e Ordenamento do Território - IGOT. Universidade de Lisboa: Lisboa, 2019.

ARAUJO, H.; RODRIGUES, M. L.; LOBO, H. Espeleoturismo em Portugal: panorama geral do uso turístico das cavidades naturais. Rosa dos Ventos, v.
9, n. $\quad 1, \quad$ p. $\quad 92-106, \quad 2017$. https://doi.org/10.18226/21789061.v9i1p092

BRASIL. Lei ${ }^{\circ} \mathbf{9 . 9 8 5}$, de 18 de julho de 2000. Institui o Sistema Nacional de Unidades de Conservação da Natureza. Diário Oficial da República Federativa do Brasil, Brasília, DF, 19 de jul. de 2000.

BOGGIANI, P. C.; SALLUN FILHO, W.; KARMANN, I.; GESICKI, A. L. D.; PHILADELPHI, N. M.; PHILADELPHI, M. Gruta do Lago Azul, Bonito, MS: onde a luz do sol se torna azul. In: Sítios geológicos e paleontológicos do Brasil. v. 2. 2009. p. $57-68$.

BOGGIANI, P. C. EIA-RIMA. Estudo de impacto ambiental e relatório de impacto ambiental e plano de manejo espeleológico da Gruta do Lago AzulMonumento natural Gruta do Lago Azul, Bonito MS. 2007.

BOGGIANI, P.C.; CLEMENTE, J. A questão do Licenciamento Ambiental de Empreendimentos Turísticos no Planalto da Bodoquena - Mato Grosso do Sul. Revista de Geografia, UFMS, AGBDourados, v. 9, p. 24- 32, 1999.

BURSZTYN, M. A.; BURSZTYN, M. Fundamentos de política e gestão ambiental. Rio de Janeiro: Garamond, 2013. 572p.

CIGNA, A.; FORTI, P. Caves: the most important geotouristic feature in the world. Tourism and Karst Areas, v. 6, n. 1, p. 9-26, 2013.

CORDEIRO, L. M.; BORGHEZAN R.; TRAJANO E. Distribuição, riqueza e conservação dos peixes troglóbios da Serra da Bodoquena, MS (Teleostei: Siluriformes). Revista da Biologia, v. 10, n. 2, p. 2127, 2013. https://doi.org/10.7594/revbio.10.02.04

CNUC. Cadastro Nacional de Unidades de Conservação. Painel das Unidades de Conservação Brasileiras. Disponível em: <http://www.mma.gov.br/areas-protegidas/cadastronacional-de-ucs>. Acesso: 25 jul. 2020.

DUTRA, A. R. S. Turismo, Áreas Protegidas e Inclusão Social: diálogos entre saberes e fazeres. Politica \& Trabalho, n. 47, p. 271-278, 2017. https://doi.org/10.22478/ufpb.1517-

$5901.2017 \mathrm{v} 1 \mathrm{n} 47.31876$

GEORGE, D.; MALLERY, P. SPSS for Windows step by step: A simple guide and reference. 11.0 update (4th ed.). Boston: Allyn \& Bacon, 2003.

GODOY, L.; LEUZINGER, M. O financiamento do Sistema Nacional de Unidades de Conservação no Brasil: características e tendências. Revista de informação legislativa, Brasília, v. 52, n. 206, p. 223$243,2015$.

GRECHI, D. C.; LOBO, H. A. S.; MARTINS, P. C. S.; LUNAS, J. R. S. Autogestão e controle de visitantes: Voucher Unificado em Bonito, MS. In: PHILIPPI JR, A.; RUSCHMANN, D. V. M. (Ed). Gestão Ambiental e Sustentabilidade no Turismo. Coleção Ambiental. V. 9, Barueri, SP: Editora Manole, 2010. p. 913-931. HORA, H. R. M.; MONTEIRO G. T. R.; ARICA J. Confiabilidade em questionários para qualidade: um estudo com o Coeficiente Alfa de Cronbach. Produto \& Produção, v. 11, n. 2, p. 85 - 103, 2010. https://doi.org/10.22456/1983-8026.9321

HOCKINGS, M.; LEVERINGTON, F.; COOK, C. Efectividad del Manejo de Áreas Protegidas. In. 
WORBOYS, G. L.; LOCKWOOD, M.; KOTHARI, A.; FEARY, S.; PULSFORD, I. Gobernanza y gestión de áreas protegidas. ANU Press, 2019. p. 949.961.

KLEIN, F. M.; ESCANDOLHERO, J. P.; LUCCHESE, N. R.; MERCANTE, M. A.; FÁVERO, S.; RODRIGUES, S. C. Educação ambiental e o ecoturismo na Serra da Bodoquena em Mato Grosso do Sul. Sociedade \& Natureza, v. 23, n. 2, p. 311-321, 2011. https://doi.org/10.1590/S198245132011000200013

KO, T. G. Development of a tourism sustainability assessment procedure: a conceptual approach. Tourism Management, v. 26, n. 3, p. 431-445, 2005. https://doi.org/10.1016/j.tourman.2003.12.003

LOBO, H. A. S.; TRAVASSOS, L. E. Cave tourism in Brazil: general aspects and its trends from the begining of the 21th century. ACKMA Journal, v. 93, p. 6 - 14.2013.

LOBO, H. A.; BOGGIANI, P. C. Cavernas como patrimônio geológico. Boletim Paranaense de Geociências, Curitiba, v. 70, p.190-199. 2013. http://dx.doi.org/10.5380/geo.v70i0.31698

LOBO, H. A. S. Fundamentos básicos do espeleoturismo. Dourados: UEMS, 2014.

LOBO, H. A. S. Characterization and management trends of negative and positive impacts of tourism in show caves. Revista Brasileira de Pesquisa em Turismo, v. 9, n. 2, p. 321-341, 2015.

MAMEDE, S. Diagnóstico e Mapeamento das Ações de Comunicação e Educação Ambiental no Âmbito do SNUC. Ministério do Meio Ambiente, Instituto Chico Mendes de Conservação da Biodiversidade, 2008. 124p.

MATHEUS, F. S.; RAIMUNDO, S. Public use and Ecotourism Policies in Brazilian and Canadian Protected Areas. Études Caribéennes, n. 33, p. 1-12, 2016.

https://doi.org/10.4000/etudescaribeennes.9344

MORETTI, E. C.; CHÁVEZ, E. S.; RIBEIRO, A. F. N. El Ecoturismo en Áreas Kársticas Tropicales: Parque Nacional Sierra da Bodoquena, Mato Grosso do Sul, Brasil y Parque Nacional Viñales, Pinar Del Rio, Cuba. Gran Tour: Revista de Investigaciones, n.13, p. 82-104, 2016.

OMT, Organización Mundial del Turismo. Indicadores de desarrollo sostenible para los destinos turísticos. Guía prática. Madrid, España: OMT, 2005. 541p.

OTEB. Observatório do Turismo e Eventos de Bonito. Desempenho da Gruta do Lago Azul. Disponível em: <http://otbonito.com.br/>. Acesso: 10 ago. 2019.

PRESCOTT-ALLEN, R. Barometer of sustainability: Measuring and communicating wellbeing and sustainable development. In: IUCN, International Union for Conservation of Nature. An approach to assessing progress toward sustainability: Tools and training series for institutions, field teams and collaborating agencies. Gland: IUCN, 1997. p. 1-39.

RUA, M. G. Turismo e Políticas Públicas de Inclusão. In: BRASIL. Ministério do Turismo. Turismo Social: Diálogos do Turismo - Uma Viagem de Inclusão. Instituto Brasileiro de Administração Municipal Rio de Janeiro: Ibam, 2006.

SÁNCHEZ-CORTEZ, J. L.; CÁRDENAS-PINTO, V.; OCAMPOS-VALAREZO, D.; JAQUE-BONILLA, D.; QUILUMBA-DUTÁN, D.; ORTIZ-BARRIONUEVO, J.; QUINTEROS-CEVALLOS, R.A.; TOLEDOROJAS, N. Aplicación de Proceso Metodológico para el Inventario Geoturístico de Cavidades Naturales en la Provincia de Napo - Ecuador. Anuário do Instituto de Geociências, v. 40, n. 2, p. 61-73, 2017. https://doi.org/10.11137/2017_2_61_73

SALAS, J. M. C.; TETTO, A. F.; REYNEL, C. A.; SOUSA, N. J.; TRES, A.; MICALOSKI, M. M.; FLORES, G. J. O. Una metodología para evaluar el manejo del turismo en áreas naturales protegidas. Journal of Biotechnology and Biodiversity. v.7, n.4, p. $\quad 413-423 \quad 2019$. https://doi.org/10.20873/jbb.uft.cemaf.v7n4.salas

SECTUR. Secretaria de Turismo, Indústria e Comércio de Bonito. Relatório da visitação da Gruta do Lago Azul -2020. Disponível em: <https://www. turismo.bonito.ms.gov.br/seictur/>. Acesso em: 10 jun. 2020.

TELES, R. M. S.; NOGUEIRA, S. M. B. Influência de programas mundiais na sustentabilidade do turismo em áreas protegidas da região metropolitana da Baixada Santista (SP). Revista Brasileira de Ecoturismo, v. 9, n.1, p. 52-173. 2016. https://doi.org/10.34024/rbecotur.2016.v9.6517

TOFETI, A. R.; DE OLIVEIRA CAMPOS, N. L. Unidades de Conservação e o território no Brasil: estudos de caso em quatro biomas. Sociedade \& Natureza, Uberlândia, MG, v.31, p.1-23, 2019. https://doi.org/10.14393/SN-v31-2019-46987

VAN BELLEN, H. M. Indicadores de Sustentabilidade: uma análise comparativa. Rio de Janeiro: Editora FGV, 2006. 235p.

\section{CONTRIBUIÇÃO DOS AUTORES}

Marta Regina da Silva-Melo concebeu o estudo, coletou e analisou os dados, redigiu e revisou o texto. Neiva Maria Robaldo Guedes orientou a elaboração do estudo e analisou os dados. Gleidson André Pereira de Melo elaborou o mapa de situação, realizou os testes estatísticos e revisou o texto. 